British Journal of Psychiatry (1991), 158, 569-578

\title{
Correspondence
}

Editor: Ian Pullen

Contents: Vasopressin in polydipsia/Psychotic depression presenting as status epilepticus/Recording of information in case notes/Motor disorders of the mentally handicapped/Engagement of relatives in intervention programmes/Patient data in child psychiatry/Child psychiatry liaison services/Beyond pumpkin seeds/Blinding trials/Demand for psychogeriatric services/How old are the elderly?/The 'new cross-cultural psychiatry'/Informed consent in India/Clozapine-treated NMS/All-night television can damage your health/Toluene-related psychosis.

\section{Vasopressin in polydipsia}

SIR: Delva et al, in their paper on vasopressin (AVP) secretion and action in polydipsic chronic psychiatric patients (Journal, November 1990, 157, 703-712), are unable to reproduce our findings that these patients (a) maintain the normal linear relationship between plasma vasopressin and plasma osmolality but exhibit a lower set point for vasopressin release, and (b) exhibit an apparently enhanced renal sensitivity to AVP's action (Goldman et al, 1988). Instead they find (a) no correlation between these patients' inappropriately elevated plasma AVP and concurrent plasma osmolality (Type I syndrome of inappropriate antidiuretic hormone (SIADH)) and (b) a normal to depressed renal sensitivity to AVP. While the authors allude to differences in method between the two studies, I believe they do not appreciate how these differences limit the ability to interpret their data.

The authors determine the relationship between plasma vasopressin and plasma osmolality by measuring these variables over several days in a naturalistic design; patients drank freely, moved freely, and smoked freely except for the hour prior to blood drawing. They compared these results with those obtained in normals using the standard controlled assessment of vasopressin secretion (oral water load (usually $20 \mathrm{cc} / \mathrm{kg}$ )) followed by urine collection every 30 minutes for four to five hours, and/or a two-hour regulated infusion of hypertonic saline (patient is kept supine, while smoking and food intake is carefully restricted). The authors do not point out that data collected by these two disparate methods may not be (and in my opinion are not) comparable. Specifically, neither the authors nor others have provided data showing that normals studied in a similar 'naturalistic' manner still exhibit a linear correlation between plasma vasopressin and plasma osmolality. Furthermore, recent data show that the effects of smoking on vasopressin function are apparent for at least 90 minutes (Allon et al, 1990), suggesting Delva et al's 60-minute smoking restriction is inadequate.

The authors use data obtained using the same basic design to assess renal sensitivity to AVP's actions. Besides the previously mentioned limitations, the authors utilised our data to define this range of normal, although we use different assays, and thus would be expected to generate different ranges. I believe their results are uninterpretable unless they define the normal range with their own assay using their design (pooled four-hour urines). I also believe pooling samples for four hours significantly reduces the sensitivity of this measure.

Finally, the authors suggest that the apparent renal hypersensitivity to AVP in our patients was due to polydipsia-induced incomplete bladder emptying. While we cannot exclude this possibility (although we have no proof of bladder dysfunction in any of the subjects), Emsley et al (1989) reported findings similar to ours using the standard controlled assessment in drug-free non-polydipsic schizophrenics. Hence other factors, currently unknown, are more likely to account for this finding.

University of Chicago Medical Center

MORRIS B. GOLDMAN 5841 South Maryland, Box 411

Chicago, IL USA 60637

\section{References}

Allon, M., Allen, H. M., Deck, L. V., et al. (1990) Role of cigarette use in hyponatremia in schizophrenic patients. American Journal of Psychiatry, 147, 1075-1077.

EmSLEY, R., Potgieter, A., TALjAAR, F. et al (1989) Water excretion and plasma vasopressin in psychotic disorders. American Journal of Psychiatry, 146, 250-253.

Goldman, M. B., Luchins, D. J. \& Robertson, G. L. (1988) Mechanisms of altered water metabolism in psychotic patients with polydipsia and hyponatremia. New England Journal of Medicine, 328, 397-403. 